\title{
Antimicrobial resistance management in Pacific Island countries: Current status, challenges, and strategic solutions
}

\author{
Royford Magiri1,2(D), Sharon Gaundan ${ }^{(1 D}$, Kennedy Choongo ${ }^{1,3}$ (D), Titus Zindove ${ }^{1}$ (D), Archibold Bakare ${ }^{1}$ (D),



\begin{abstract}
1. Department of Veterinary Science, College of Agriculture, Fisheries and Forestry, Fiji National University, Suva P.O. Box 7222, Fiji; 2. School of Public Health, University of Saskatchewan, Saskatoon, SK, Canada; 3. Department of Veterinary Biomedical Sciences, School of Veterinary Medicine, University of Zambia, P. O. Box 32379, Lusaka, Zambia;

4. Department of Public Health, College of Medicine, Nursing and Health Sciences, Fiji National University, Private

Mail Bag, Hoodless House Campus, Brown Street, Suva, Fiji; 5. Commonwealth Scientific and Industrial Research Organization, Black Mountain Science and Innovation Park, Clunies Ross Street, Acton ACT 260, Australia; 6. Ministry of Health and Medical Services, Dinem House, 88 Amy St, Suva, Fiji.

Corresponding author: Royford Magiri, e-mail: roybundimag@gmail.com

Co-authors: SG: gaundan.s.sharon@gmail.com, KC: kennedychoongo@yahoo.ie, TZ: zindovetj@gmail.com,

AB: archibold.bakare@fnu.ac.fj, EO: euniceadoma@yahoo.co.uk, WO: walter.okelo@csiro.au, GM: george.mutwiri@usask.ca, ER: eric.rafai@govnet.gov.fj, AG: ashodra.gautam@govnet.gov.fj, PI: pauladeiji@gmail.com

Received: 22-08-2021, Accepted: 09-12-2021, Published online: 19-02-2022
\end{abstract}

doi: www.doi.org/10.14202/IJOH.2022.1-7 How to cite this article: Magiri R, Gaundan S, Choongo K, Zindove T, Bakare A, Okyere E, Okello W, Mutwiri G, Rafai E, Gautam A, Iji P (2022) Antimicrobial resistance management in Pacific Island countries: Current status, challenges, and strategic solutions, Int J One Health, 8(1): 1-7.

\begin{abstract}
Antimicrobial resistance (AMR) is currently recognized as a major emerging threat to human and animal health. The burden of antimicrobial-resistant infections affects the economy in developed and developing countries. There is a rapid rise in AMR in human and veterinary medicine globally. AMR profiles are poorly documented in Fiji, and limited data are accessible. Fiji currently has no national veterinary antibiotic resistance surveillance network or regulations and guidelines on veterinary drug use. However, available literature shows that although human drugs are better managed than veterinary drugs, the knowledge is still constrained and dispersed. Furthermore, Fiji was chosen as a case study to develop a prototype AMR surveillance and control in the Pacific region. Pacific Island countries share similar geographic and climatic conditions. Currently, the Australian Centre for International Agricultural Research has funded an AMR project that addresses some gaps in managing AMR in the region. The project is the first to adopt the One Health approach to research the AMR in humans, animals, and the Pacific region's environment. Combating AMR needs human health and veterinary personnel to work with all other stakeholders. Continuous surveillance for resistant clinical isolates in humans and animals and the development of appropriate policy intervention measures in human and veterinary drug use are necessary to alleviate AMR burden. Therefore, there is a need to educate farmers, human patients, and the public on the fight against AMR. In addition, AMR data are necessary to develop effective AMR control strategies. This review gives a comprehensive information assessment on AMR in Fiji and the other South Pacific Islands in relation to global trends. Suggestions on the most appropriate ways of effectively managing AMR in Fiji have been made.
\end{abstract}

Keywords: Antimicrobial resistance, Pacific Island countries, strategic management.

\section{Introduction}

Antimicrobial resistance (AMR) is recognized as one of the most urgent rising threats to global human and animal health systems, especially in developing countries [1]. A microorganism can evade the effects of an antibiotic [2]. Antibiotics are either cytostatic, allowing the natural immune system to kill the bacteria, or cytotoxic and directly kill the bacteria [3]. Antibiotics act through different mechanisms, such as inhibition by bacterial cell wall synthesis, enhanced protein denaturation, inhibition of deoxyribonucleic acid replication or segregation, or both, the transcription process of ribonucleic

Copyright: Magiri, et al. This article is an open access article distributed under the terms of the Creative Commons Attribution 4.0 International License (http://creativecommons.org/licenses/ by/4.0/), which permits unrestricted use, distribution, and reproduction in any medium, provided you give appropriate credit to the original author(s) and the source, provide a link to the Creative Commons license, and indicate if changes were made. The Creative Commons Public Domain Dedication waiver (http:// creativecommons.org/ publicdomain/zero/1.0/) applies to the data made available in this article, unless otherwise stated. acid, and disintegration of the cell membrane [4]. Antibiotics may enter the microorganisms through cell wall attachments, energy-dependent transport mechanisms, or cross-membrane transport systems [5]. Different antibiotic agents have been used for therapeutic purposes over a long time, and resistance has been reported when the recommended drug dose becomes ineffective against the bacteria. The bacteria would be unaffected by the drug because they would have developed resistance; thus, they would continue to multiply [3].

AMR results in the development of "superbugs" whose infections are untreatable using standard antibiotic therapy $[6,7]$. It has been projected that deaths because of AMR-related bacterial infections will increase exponentially from 700,000 in 2015 to 10 million deaths by 2050 [8]. The World Bank estimates that AMR will decrease global gross domestic product (GDP) by $1-4 \%$ by 2050 and global livestock production by $2.5-7.5 \%[9,10]$. Without effective antibiotics, disease treatment will be unsuccessful, 
causing premature human mortality and decreased contribution of animal products to food security and livelihoods.

It is currently projected that AMR could cost the Western Pacific Region as much as USD 1.35 trillion by 2032 [11]. AMR can spread regionally and globally; it is a global public health threat, so all countries must pool resources to mitigate it, irrespective of their AMR status. A study by Jenney et al. [12] in Fiji hospitals reported 50 cases of Staphylococcus aureus/100,000 people, one of the highest bacterial infection rates worldwide. AMR may be the main contributor to the high rate of bacterial infections in Fiji [12]. In Fiji, there are more cases of human diabetes, with amputation rates of one every $12 \mathrm{~h}$, and tuberculosis in human beings and animals [13-15]. High incidence of bacterial infections and surgery for complications, such as diabetes, needing post-surgical antibiotic drugs for managing infected wounds increases AMR incidence. Besides human beings, Antimicrobial-resistant microbes are also found in food, animals, water, soil, and air [16]. Therefore, AMR can be spread between people and animals through the food of animal and plant origin, water, and air. Antimicrobials in livestock have been widely reported as the main contributor to the emergence and spread of AMR, especially in developing countries, where there is an imprudent use of antimicrobial agents in the livestock production sector [1719]. Because antimicrobial resistant organisms can be transferred from humans to animals directly or through the environment, or both, and vice versa, strategies to reduce the emergence and spread of AMR should take a holistic and multisector approach, which encompasses humans, animals, and their environment. This approach is commonly referred to as One Health. Fiji currently lacks a government policy for veterinary AMR surveillance networks and regulations and guidelines on the proper use of veterinary drugs. In addition, there is a lack of data on AMR profiles in animals. This lack of well-documented information on AMR is a barrier to the development of effective AMR control strategies, hence the relevance of this review to discuss AMR in Fiji, Pacific Island countries, and the possible mitigation strategies.

\section{Epidemiology of AMR in Fiji}

Antimicrobial-resistant organisms can be transferred from humans to animals directly or through the environment, or both. Therefore, strategies to reduce the emergence and spread of AMR should take a holistic and multisector approach that encompasses human beings, animals, and their environment. Limited studies, to date, have examined the epidemiology of AMR in the Pacific, particularly in Fiji [12]. This makes AMR a significant threat to public health in Fiji. However, no study linking humans, animals, and the environment to AMR in Fiji necessitates investigations.

\section{Global AMR Crisis}

A huge number of various antibiotic classes have been produced in the past seven decades since the discovery of antibiotics [3]. Increased demand across many sectors of society and the reduced cost of antibiotics have promoted these drugs' off-label uses [20]. This extensive application of antibiotics has caused the emergence of resistant strains of infectious organisms [3]. Previously, new antibiotic drugs were developed to combat any emerging resistant organisms proportionately. However, nowadays, the general approach in fighting against emerging and reemerging resistant microorganisms is based on the modification of existing antibiotics [21]. Interestingly, no new class of antibiotics has been discovered in the last four decades since 1962-2000 when nalidixic acid and linezolid were introduced. Thus, the antibiotics in the market during that period resulted from modifying existing molecules [22]. Developing new antimicrobial agents is costly and time-consuming, resulting in reduced interest by pharmaceutical industries [23].

The demonstration of resistance to antibiotics developing within a short time is a matter of concern [3]. In developing countries, most antibiotics are sold over-the-counter or without prescription, or both, a major contributing factor in resistance development $[24,25]$. Therefore, it is essential to educate all stakeholders, including farmers, patients, and the public, to combat antibiotic resistance. In addition, the national veterinary antibiotics resistance surveillance network and regulations and guidelines on veterinary drug use will be effective in combating AMR.

\section{General mechanisms of the development of antibiotic resistance}

Antibiotics kill or inhibit the normal growth of microorganisms through different mechanisms of action. Hence, antibiotic pressure forces these organisms to develop or engage their natural survival process, thereby driving or encouraging the development of resistance. The resistance process through gene mutation level induced by selection pressure occurs due to the exchange of resistant genes between humans, animals, and the environment [26]. Natural selection is not the only mechanism of evolution of resistance because bacteria can directly transfer genetic material through plasmids. Therefore, intensive antibiotic use simply accelerates the process. Prescription of broad-spectrum antibiotics in hospitals following nosocomial infections accelerates resistance by increasing selection pressure [3]. Antibiotics could remove major susceptible bacteria, but few non-susceptible bacteria that could have genetically mutated resistance to antibiotics would continue growing [3].

Moreover, antibiotic-resistant infection levels are associated with the magnitude of antibiotic consumption [3]. Failure to take prescribed antibiotic therapy fully is likely to induce resistance. The bacteria that would need a complete course to be eliminated 
subsequently survive while gaining more strength against antimicrobial attack [3]. Furthermore, with time, bacteria can acquire many resistance traits and become resistant to many classes of antibiotics [27]. Particularly, the resistance observed in staphylococcal chromosomal mutations is due to ineffective transport of aminoglycosides into the bacteria in addition to enzyme modification [28]. Moreover, organisms resistant to one antibiotic may become resistant to other antibiotics that are similar in structure and from the same class. For example, tetracycline resistance may have cross-resistance to other related compounds such as oxytetracycline, chlortetracycline, doxycycline, and minocycline [3].

\section{Antibiotics for growth promotion in animals}

For several decades, antibiotics have been used to enhance growth and increase livestock production, mostly in poultry and swine [29]. Antibiotics improve growth, feed efficiency, and animal health by acting on the gastrointestinal bacteria of the animal [30]. The use of AGMP encourages lethal or significant pathogen damage, causing reduced bacterial toxin production. It further reduces the use of essential nutrients by gut microorganisms, thus increasing the synthesis and availability of vitamins and other growth factors in livestock [30]. However, the abuse of antibiotics as growth promoters in livestock and food production has widely contributed to the alarming increase in global AMR concerns [29,31]. Human health can be either directly influenced through residues of antibiotics in meat, which may cause reactions, or indirectly through the resolution of antimicrobial residues that may spread to microorganisms in humans [31]. AMR emergence has mainly been attributed to the abuse or misuse, or both, of antimicrobials as therapeutic drugs in human and animal health care or as antibiotic growth promoters in the animal husbandry sector [25]. This AMR has been attributed to selective pressure and acquired through direct transfer of multidrug-resistant (MDR) organisms at the human, animal, and environment interface [30]. There are many reports of increased resistance due to antimicrobial growth promoters $[29,32]$. In addition, the transmission of MDR organisms can occur through direct person-to-person contact, animal-to-person contact (scratches and bites), contact with livestock through the food chain, and contact with waste from farms [33]. Evidence suggests the transfer of antimicrobial-resistant bacteria from humans through food and direct contact [34].

In 1986, Sweden became the first nation to eliminate the antimicrobials used for food animal production and growth promotion [29]. Denmark and other European Union countries followed Sweden's example and put forth a legislative ban on AGMP. Before the ban on AGMP in Denmark, the total usage of antimicrobials in food animals was reportedly $205,686 \mathrm{~kg}$ annually. A reduction of $54 \%$ was noted in 2001 with 94,200 kg post-ban legislation [29]. There is no information on antibiotic use in animal feeds, regulations, and guidelines in Fiji and the rest of the Pacific region. Further, AMR patterns are poorly documented, and there is no information on bacterial prevalence and antibiotic sensitivity patterns. This needs further investigation and the establishment of government-level veterinary and human antimicrobial use and resistance surveillance network.

\section{Therapeutic antimicrobials used in animals and humans}

Many pathogenic and nonpathogenic bacteria originate from food animals to humans [35]. The presence of such organisms in the same host enhances environmental interactions, resulting in the evolution of new drug-resistant and MDR bacteria due to the horizontal exchange of drug-resistant genes [35]. The excessive application of antibiotics in livestock for growth and disease control is a trigger for developing drug-resistant strains [1]. The indiscriminate use of antimicrobials in human medicine is the other trigger [36]. Antimicrobial therapeutic and prophylactic use against infectious diseases is widely used in human and animal health sectors [35]. Microorganisms resistant to one particular antimicrobial agent can develop resistance to others with similar pharmacologically active agents resulting in MDR organisms [36].

This use of antimicrobials is well documented in developed countries. However, it is poorly documented in developing countries, including Fiji, and if documented, the information is not readily available. The Union of Concerned Scientists has estimated antibiotic use in the human health sector as 1.36-14.64 million $\mathrm{kg} /$ year, whereas the estimated use of antimicrobials in agriculture and livestock, is 7.36-11.18 million $\mathrm{kg} /$ year [30].

\section{The economic impact of AMR in the developing countries}

In 2014, the first surveillance report of the World Health Organization (WHO) on AMR stated that five of six WHO regions had above 50\% resistance to the third-generation cephalosporin and fluoroquinolones in Escherichia coli and methicillin resistance in $S$. aureus in a clinical setting (WHO, 2014). One of the many consequences of AMR is its economic burden [37]. When a patient is unresponsive to antimicrobial treatment because of AMR, the patient remains sick longer than a patient without resistance problems. This person would require prolonged hospitalization treatment with expensive and toxic drugs, increasing the health-care system's burden [37]. According to Song, the overall societal costs for the detection, treatment, management, and surveillance of AMR are approximately \$US35 million in the United States alone, 1.5 billion Pound Sterling (GBP) in the European Union, and \$US1.3 billion in Thailand [38]. The author further showed that low- and middle-income countries would have a greater burden due to more significant abuse of antibiotics and a lack of 
data on AMR. Therefore, the overall global impact of AMR in the economic aspects is unprecedented to date. However, in its AMR Report 2020, World Bank projected that in the case of low AMR impacts, the annual global GDP is likely to reduce by $1.1 \%$ by the year 2050.

Similarly, the world is expected to lose $3.8 \%$ of its yearly GDP by 2050 in a high AMR impact scenario. This could cause an annual shortfall of \$US3.4 trillion by 2030 [9]. Ordinarily, AMR is discussed as a threat to human health; it is, however, a complex problem associated with livestock and environmental health [39]. AMR emerged because of the bacterial exposure to antibiotics in medicine, crop production, households, and farms. Because of the decline in the effectiveness of existing antibiotic agents, infections have become challenging and expensive to treat [36]. Furthermore, this also threatens the control of infectious diseases and epidemics [36].

AMR poses a higher threat for developing countries like Fiji due to the lack of proper surveillance and inadequate infrastructure for monitoring and AMR management. Another factor leading to the emergence of AMR is the administration of broad-spectrum antibiotics as an empirical therapy to outpatients [37]. Intensifying this problem is the lack of consumer and public knowledge regarding the proper use of antibiotics. Self-medication and poor patient compliance are other factors contributing to the rapid emergence of AMR [34,37]. Kumar et al. [37] further suggested that the lack of national commitments and ill-defined accountability in antimicrobial use is the leading cause of recent increasing resistance to antimicrobials. A comprehensive, well-coordinated response and ill-defined accountability in terms of antimicrobial use and resistance in addition to national surveillance and monitoring systems in human and animal healthcare sectors are of paramount importance in detecting AMR and its emergence.

\section{AMR Burden in the Pacific Island States}

The Pacific Island Countries and Territories (PICTs) comprise 22 members of the Secretariat of the Pacific Community. The World Bank defines these countries as low- and middle-income countries with key industries that include agriculture, aquaculture, tourism, and forestry. Loftus et al. [39] further explained the lack of literature on the burden of AMR in the PICTs. Fiji, New Caledonia, and Papua New Guinea are the three countries with over $30 \%$ of the limited published information on AMR in this region. Furthermore, there is no information on the rates of antimicrobial use in livestock in the PICTs. Of the 22 countries, only five PICTs are members of the World Organization for Animal Health [40]. The small size of the PICTs, limited resources, substandard health facilities/infrastructures, and natural disasters like cyclone susceptibility, make them vulnerable to AMR effects [40].
Between 2011 and 2017, Fiji reported25\% and $0.7 \%$ of Klebsiella pneumoniae (KPN) resistance to cephalosporin and carbapenems, respectively [39]. There was no information on KPN resistance to fluoroquinolone chloramphenicol, tetracycline, aminoglycoside, and penicillin. In more recent years, there is a scope for further research on antibiotic-resistant (ABR) KPN. A detailed investigation is needed to analyze ABR yearly.

Between 2011 and 2017, some ABR E. coli was reported in Fiji. Penicillin-resistant $E$. coli was found to be $85 \%$ during this period, and fluoroquinolone and cephalosporin resistance was at $11.9 \%$ and $12.2 \%$, respectively [39]. No data were available for chloramphenicol- and aminoglycoside-resistant $E$. coli.

In Fiji, AMR surveillance for Neisseria gonorrhoeae has strengthened over the years, which is evident from the information on the ABR $N$. gonorrhoeae $[39,40]$. Penicillin-resistant $N$. gonorrhoeae was reported to be $8.3 \%$ between 2000 and 2010 . However, the prevalence of penicillin-resistant $N$. gonorrhoeae reduced between 2011 and 2017. In contrast, a notable increase in the prevalence of fluoroquinolone resistance was evident. Between 2000 and 2010, the prevalence of $N$. gonorrhoeae resistance to fluoroquinolone was a mere $0.6 \%$, whereas there was an increase of $N$. gonorrhoeae resistance to fluoroquinolone by 6\% between 2011 and 2017 [40]. No information was available on the cephalosporin-resistant $N$. gonorrhoeae between 2000 and 2010. However, a $0.4 \%$ prevalence of cephalosporin-resistant $N$. gonorrhoeae was reported in 2011 and 2017 [40].

\section{Regulatory Issues Related to Antibiotic Resistance}

There are well-established international guidelines for managing daily antibiotic practices. However, these regulatory guidelines vary between nations [41]. Nations such as the United Kingdom and Australia have acted swiftly to offer guidance, but many countries, including PICTs, have not developed the regulatory guidelines. In addition, WHO has developed guidelines for limited antibiotic use in children, indicating antibiotic use in this group for severe bloody diarrhea and cholera [42]. Many organic and inorganic toxins have been released into streams, rivers, oceans, land, and air as industrial waste and this may enhance transfer of antibiotic resistant genes [43]. Furthermore, insufficient guidelines for disposal of home hygiene products may also present a major resistance risk because a high number of antibacterial substances are found in such products [43].

Evidence suggests that AMR is more prevalent where an enormous amount of antibiotics is used; hence, global antibiotic use cannot be ignored [43]. The absence of guidelines and regulations on antibiotics is high among low-income countries. The major problems contributing to the greatest resistance burden in developing countries are low antibiotic prices, 
ease of general availability, and inappropriate antibiotic use [25]. In countries with limited universal health policy to the general populace, antibiotic use is generally not well controlled, making inappropriate use of antibiotics one of the greatest concerns [3].

Everyone is affected by AMR; thus, it is a major public health concern affecting several sectors from clinical personnel in either human or veterinary practice, specialists in the pharmaceutical industry, and the public generally [3]. Therefore, this needs a multi-sectoral approach encompassing all industry stakeholders in combating this eminent public health burden. The application of modern susceptibility tests ensures that humans and animals take antibiotics on time [3]. Moreover, the use of antibiotics in agricultural production needs to be regulated in the PICTs.

The use of antibiotics as a therapy for bacterial infections is the major method to control infections. However, infections may continuously persist in cases of resistance to antibiotics. The overall threat is the increasing demand for new and effective antibiotics whose resistance has not yet occurred.

\section{Antimicrobial Use and Link to Bacterial Resistance in the Pacific}

Antibiotic use, misuse, and abuse in human and veterinary practice exert the greatest selection pressure for AMR development in the PICTs. There is a paucity of information regarding antibiotic use and resistance in human and veterinary practice in the Pacific. Furthermore, there are minimal efforts to determine the quantities of antimicrobials used in livestock, and having information on quantities of antimicrobial use in animals is essential in determining the key drivers for developing antibiotic resistance in the Pacific. Increased AMR in human pathogens that spread resistance from the closed hospital environment into open communities is another threat to public health in the Pacific. Increased use of antimicrobials in livestock is the primary cause of AMR development. This information is limited in the Pacific and needs further investigation in Fiji.

\section{Strategies for Resistance Control in the $21^{\text {st }}$ Century}

Global action plan on antimicrobial resistance was developed by the WHO and OIE. It was adopted and endorsed by the World Health Assembly in May 2015 to tackle the problem of AMR [44]. GAP outlines five specific strategies to combat AMR. The first strategic objective is to improve knowledge on AMR. The second is strengthening knowledge by intensifying surveillance and research. The third and fourth strategic objectives are to decrease the infection rate and optimize the antimicrobial agents' use. The final strategic objective is to develop a sustainable investment economic model that includes all countries and increases investment in new medicine, diagnostic tools, vaccines, and other interventions in combating
AMR. GAP calls upon all nations to design national action plans (NAPs) in line with world strategies [39]. Fiji became the first Pacific Island country to develop and launch a NAP for AMR. Fiji is now one of the two PICTs that have NAPs developed and approved by the government [39]. However, AMR surveillance in Fiji is hindered mainlyby limited access to necessary equipment, expertise, and materials. Although there are data for human health, these are rarely linked to a centralized system or other private facilities. In addition, laboratory surveillance for animal health is virtually non-existent. This is evident from the country situational report conducted before the NAP launch, which identified that the national antimicrobial response committee included all stakeholders except the veterinary sector.

To combat the problem of antibiotic abuse, Fiji, one of eight PICTs, has developed antimicrobial usage guidelines. The standard treatment guideline application created by the Australian therapeutic guidelines foundation has been madefreely and easily accessible to smartphone users in Fiji and the Solomon Islands [39]. The regional synthesis report and recommendations of 2018 state that no country has developed a total capacity to prevent, detect, and treat the emerging AMR threat [45]. However, it was further reported that Fiji has the most stable system and is well equipped to provide support to other countries in the region if needed.

\section{Conclusion}

Antibiotics are necessary to combat pathogenic infections. Over the years, several different classes of antibiotics have been used for therapeutic purposes and prophylactically in numerous fields like agriculture to increase production. This has led to the development and emergence of resistance. This review observes that there is limited knowledge on AMR in the Pacific countries, which is an alarming issue. Notably, in the Pacific and Fiji, some infections, such as pneumonia, tuberculosis, and gonorrhea, are increasingly more challenging to treat due to less effective antibiotics. Antibiotic-resistant infections may be directly linked to quantities and levels of use. This information is lacking in Fiji and needs further investigation. In addition, inappropriate antibiotic use may be associated with AMR in Fiji and the Pacific. However, there are limited studies to establish the knowledge, attitude, and practices regarding antibiotic use in the Pacific. Also, in Fiji, there is limited knowledge regarding antibiotic resistance in the population. It is essential to educate patients, farmers, and the public about the dangers posed by AMR; knowledge of the resistance pattern is essential. Moreover, antibiotic use in livestock is increasing at an alarming rate globally, despite the WHO and OIE recommendations. There is a need for antibiotic use surveillance and reporting system to aid in adhering to global AMR management policies in Fiji. 


\section{Authors' Contributions}

RM: Conceived the idea, supervised the drafting of the manuscript. SG: Prepared the original draft of the manuscript. $\mathrm{KC}, \mathrm{TZ}$, and $\mathrm{AB}$ : Edited the original draft of the manuscript. EO, GM, and PI: Substantively revised the manuscript. WO: Edited the manuscript and provided resources for publication. ER and AG: Contributed to the human aspect of the review. All authors read and approved the final manuscript.

\section{Acknowledgments}

The authors acknowledge the support of Fiji National University by Commonwealth Scientific and Industrial Research Organization to enhance the management of AMR in Fiji. The study was financially supported by Commonwealth Scientific and Industrial Research Organization, Australia (Grant number-LS/2019/119).

\section{Competing Interests} interests.

The authors declare that they have no competing

\section{Publisher's Note}

Veterinary World (Publisher of International Journal of One Health) remains neutral with regard to jurisdictional claims in published institutional affiliation.

\section{References}

1. Prestinaci, F., Pezzotti, P. and Pantosti, A. (2015) Antimicrobial resistance: A global multifaceted phenomenon. Pathog. Glob. Health, 109(7): 309-318.

2. Mukerji, S., O’Dea, M., Barton, M., Kirkwood, R., Lee, T. and Abraham, S. (2017) Development and transmission of antimicrobial resistance among Gram-negative bacteria in animals and their public health impact. Essays Biochem., 61(1): 23-35.

3. Zaman, S.B., Hussain, M.A., Nye, R., Mehta, V., Mamun, K.T. and Hossain, N. (2017) A review on antibiotic resistance: Alarm bells are ringing. Cureus, 9(6): e1403.

4. Regea, G. (2018) Pharmacology and Clinical Research Review on Antibiotics Resistance and its Economic Impacts. J. Pharmacol. Clin. Res., 5(5): 1-9.

5. Chopra, I. and Roberts, M. (2001) Tetracycline antibiotics: Mode of action, applications, molecular biology, and epidemiology of bacterial resistance. Microbiol. Mol. Biol. Rev., 65(2): 232-260.

6. Adegoke, A.A., Faleye, A.C., Singh, G. and Stenström, T.A. (2017) Antibiotic-resistant superbugs: Assessment of the interrelationship of occurrence in clinical settings and environmental niches. Molecules, 22(1): 29.

7. Smith, R.D. and Coast, J. (2002) Antimicrobial resistance: A global response. Bull. World Health Organ., 80(2): 126-33.

8. de Kraker, M.E.A., Stewardson, A.J. and Harbarth, S. (2016) Will 10 million people die a year due to antimicrobial resistance by 2050? PLoS Med., 13(11): e1002184.

9. Baris, E., Jonas, O., Irwin, A., Berthe, F., Le Gall, F., Gall, F.L., Marquez, P.V., Nikolic, I., Plante, C., Scheneidman, M., Shriber, D. and Thiebaud, A. (2017) Drug-Resistant Infections: A Threat to Our Economic Future. World Bank Group, Washington, DC

10. World Health Organization. (2006) The Global Plan to Stop TB, 2006-2015, Report No. 9241593997. World
Health Organization, Geneva

11. World Health Organization. (2017) Antimicrobial Resistance in the Asia Pacific Region: A Development Agenda. WHO Regional Office for the Western Pacific, Manila.

12. Jenney, A., Holt, D., Ritika, R., Southwell, P., Pravin, S., Buadromo, E., Carapetis, J., Tong, S. and Steer, A. (2014) The clinical and molecular epidemiology of Staphylococcus aureus infections in Fiji. BMC Infect. Dis.,14(1): 1-7.

13. Phillips, T. (2020) The everyday politics of risk: Managing diabetes in Fiji. Med Anthropol., 39(8): 1-16.

14. Borja, E., Borja, L.F., Prasad, R., Tunabuna, T. and Toribio, J.A.L. (2018) A retrospective study on bovine tuberculosis in cattle on Fiji: Study findings and stakeholder responses. Front. Vet. Sci., 5: 270.

15. Ragonnet, R., Underwood, F., Doan, T., Rafai, E., Trauer, J. and McBryde, E. (2019) Strategic planning for tuberculosis control in the Republic of Fiji. Trop. Med. Infect. Dis., 4(2): 71.

16. Ahmed, S., Ibrahim, M., Ahmad, F., Rana, H.A., Rao, T., Anwar, W., Afzaal, M., Mirza, S.A., Arooj, T. and Almas, M. (2020) Microbial risk assessment and antimicrobial resistance. In: Antibiotics and Antimicrobial Resistance Genes in the Environment. Elsevier, Amsterdam, Netherlands. p313-330.

17. Ayukekbong, J.A., Ntemgwa, M. and Atabe, A.N. (2017) The threat of antimicrobial resistance in developing countries: Causes and control strategies. Antimicrob. Resist. Infect. Control, 6(1): 47

18. Clement, M., Olabisi, M., David, E. and Issa, M. (2019) Veterinary pharmaceuticals and antimicrobial resistance in developing countries. In: Veterinary Medicine and Pharmaceuticals. IntechOpen, UK, London.

19. Medina, M.J., Legido-Quigley, H. and Hsu, L.Y. (2020) Antimicrobial Resistance in One Health. Global Health Security. Springer, Berlin, Germany. p209-29.

20. Kocs, D. and Fendrick, A.M. (2003) Effect of off-label use of oncology drugs on pharmaceutical costs: The rituximab experience. Am. J. Manag. Care, 9(5): 393-400.

21. Coates, A., Hu, Y., Bax, R. and Page, C. (2002) The future challenges facing the development of new antimicrobial drugs. Nat. Rev. Drug Discov., 1(11): 895-910.

22. Wright, P.M., Seiple, I.B. and Myers, A.G. (2014) The evolving role of chemical synthesis in antibacterial drug discovery. Angew. Chem. Int. Ed. Engl., 53(34): 8840-8869.

23. Birkett, D., Brøsen, K., Cascorbi, I., Gustafsson, L.L., Maxwell, S., Rago, L., Rawlins, M., Reidenberg, M., Sjiqvist, F., Smith, T., Thuerman, P., Walubo, A. and Orme, M. (2010) Clinical pharmacology in research, teaching and health care: Considerations by IUPHAR, the international union of basic and clinical pharmacology. Basic Clin. Pharmacol. Toxicol., 107(1): 531-559.

24. Okeke, I.N., Lamikanra, A. and Edelman, R. (1999) Socioeconomic and behavioral factors leading to acquired bacterial resistance to antibiotics in developing countries. Emerg. Infect. Dis., 5(1): 18.

25. Chokshi, A., Sifri, Z., Cennimo, D. and Horng, H. (2019) Global contributors to antibiotic resistance. J. Glob. Infect. Dis., 11(1): 36-42.

26. Tello, A., Austin, B. and Telfer, T.C. (2012) Selective pressure of antibiotic pollution on bacteria of importance to public health. Environ. Health Perspect., 120(8): 1100-1106.

27. Fair, R.J. and Tor, Y. (2014) Antibiotics and bacterial resistance in the $21^{\text {st }}$ century. Perspect. Medicin. Chem., 6: 25-64.

28. Munita, J.M. and Arias, C.A. (2016) Mechanisms of antibiotic resistance. Microbiol. Spectr., 4(2): 42

29. Dibner, J.J. and Richards, J.D. (2005) Antibiotic growth promoters in agriculture: History and mode of action. Poult. Sci., 84(4): 634-643.

30. Modi, C.M., Mody, S.K., Patel, H.B., Dudhatra, G.B., Avinash, K. and Sheikh, T.J. (2011) Growth promoting use 
of antimicrobial agents in animals. J. Appl. Pharm. Sci., 1(8): 33-36.

31. Graham, J.P., Boland, J.J. and Silbergeld, E. (2007) Growth promoting antibiotics in food animal production: An economic analysis. Public Health Rep., 122(1): 79-87.

32. van den Bogaard, A.E. and Stobberingh, E.E. (2000) Epidemiology of resistance to antibiotics: Links between animals and humans. Int. J. Antimicrob. Agents, 14(4): 327-335.

33. Nelson, D.W., Moore, J.E. and Rao, J.R. (2019) Antimicrobial resistance (AMR): Significance to food quality and safety. Food Qual. Saf., 3(1): 15-22.

34. Byarugaba, D.K. (2004) Antimicrobial resistance in developing countries and responsible risk factors. Int. J. Antimicrob. Agents, 24(2): 105-110.

35. Marshall, B.M. and Levy, S.B. (2011) Food animals and antimicrobials: Impacts on human health. Clin. Microbiol. Rev., 24(4): 718-733.

36. Eggleston, K., Zhang, R. and Zeckhauser, R.J. (2010) The global challenge of antimicrobial resistance: Insights from economic analysis. Int. J. Environ. Res. Public Health, 7(8): 3141-3149.

37. Kumar, S.G., Adithan, C., Harish, B.N., Sujatha, S., Roy, G. and Malini, A. (2013) Antimicrobial resistance in India: A review. J. Nat. Sci. Biol. Med., 4(2): 286-291.
38. Song, J. (2015) Antimicrobial Resistance Control in Asia, AMR Control. p40-46.

39. Loftus, M.J., Stewardson, A.J., Naidu, R., Coghlan, B., Jenney, A.W.J., Kepas, J., Lavu, E., Munamua, A.B., Peel, T.N., Sahai, V., Tekoaua, R., Tudravu, L., Zinihite, J., Cheng, A.C., Rafai, E. and Peleg, A.Y. (2020) Antimicrobial resistance in the Pacific Island countries and territories. BMJ Glob. Health., 5(4): e002418.

40. Foxlee, N.D., Townell, N., McIver, L. and Lau, C.L. (2019) Antibiotic resistance in pacific Island countries and territories: A systematic scoping review. Antibiotics (Basel), 8(1): 29.

41. Davies, J. and Davies, D. (2010) Origins and evolution of antibiotic resistance. Microbiol. Mol. Biol. Rev., 74(3): 417-433.

42. Manyi-Loh, C., Mamphweli, S., Meyer, E. and Okoh, A. (2018) Antibiotic use in agriculture and its consequential resistance in environmental sources: Potential public health implications. Molecules, 23(4): 795.

43. Ferronato, N. and Torretta, V. (2019) Waste mismanagement in developing countries: A review of global issues. Int. J. Environ. Res. Public Health, 16(6): 1060.

44. Report on Antimicrobial Resistance (AMR). (2020) FAO Regional Conference for Asia and the Pacific.

45. Jimmie, I.A.R. (2018) Condon Rob Regional Synthesis Report and Recommendations Pacific Health Security Mission.

\section{$* * * * * * * *$}

